\title{
Percepciones socioculturales de los pobladores de la comunidad el Limón ante un riesgo climático
}

\author{
Osmany Maurice Pérez Lanuza ${ }^{1}$ \\ María José Romero Arteta ${ }^{2}$ \\ Franklin Solís Zúniga ${ }^{3}$ \\ Heydi Fabiola Castillo Rodríguez ${ }^{4}$
}

\section{RESUMEN}

El presente artículo, resume una investigación cualitativa con enfoque de Investigación-Acción, realizado con jóvenes y adultos habitantes afectados por los riesgos climáticos. La investigación se llevó a cabo en la comunidad El Limón de la subzona de Santa Cruz, Estelí, en el período entre septiembre y diciembre del año 2014. Con esta se pretendía valorar las percepciones socioculturales de los pobladores ante un riesgo climático. Se realizó desde la metodología de investigación, acción participativa, dentro de las técnicas utilizadas están: la encuesta, entrevista semi-estructurada, guía de observación y grupo focal, además de la revisión documental. Las diferentes técnicas se analizaron de acuerdo a cada objetivo y a su naturaleza de IAP. Dentro de los principales hallazgos se encuentra que los pobladores no poseen suficiente conocimiento sobre riesgo, además de existir una influencia en el desarrollo de sus percepciones con respecto a su posición geográfica, ubicada cerca de urbanizadoras. Referido a los efectos que perciben en las actividades socioambientales únicamente ven evidente la escases de agua, el aumento de plagas y enfermedades. Por ello se elaboró una propuesta de acción, encaminada a fomentar la auto gestión como medida de adaptación al cambio climático.

Palabras Claves: Percepciones Socioculturales, Riesgo Climático, Actividades Socioambientales.

Recibido: 3 de marzo de 2016

Aceptado: 4 de abril de 2016

1 UNAN-Managua, FAREM-Estelí. Correo electrónico: osmany.maurice@gmail.com 2 UNAN-Managua, FAREM-Estelí. Correo electrónico: maryroart@yahoo.com

3 Docente UNAN-Managua, FAREM-Estelí. Correo electrónico: franksolis23@yahoo.com 4 UNAN-Managua, FAREM-Estelí. 


\title{
Social and cultural perception of the population of the community El Limón in a climate risk
}

\begin{abstract}
This article summarizes a qualitative research approach as an action research conducted with young and adult people affected by climate risks. The research was carried out in the community El Limón of the sub zone of Santa Cruz, Estelí, in the period between September and December 2014. This research was intended to assess the socio-cultural perceptions of people from the community in a climate risk. It was made as a methodology research, participatory action within the techniques used are: the survey, semi - structured interview, observation guide and focus group, in addition to the document review. Different techniques were analyzed according to each purpose and nature of IAP. Among the main findings it is that people do not have enough knowledge about risk also exists an influence on the development of their perceptions of their geographical position, located near urban housing developers. Referring to the effects perceived from the social and environmental activities are evident only water insufficiency, increased pests and diseases. Therefore a proposal for action, aimed at development self-management as a measure of adaptation to climate change was developed.
\end{abstract}

Keywords: Socio-Cultural Perceptions, Climate Risk, Social and Environmental Activities. 


\section{INTRODUCCIÓN}

En las últimas décadas, el ser humano ha sido testigo de la ocurrencia de fenómenos naturales y antrópicos lo cual hace pensar que hay factores importantes que en parte se han alterado y han venido cambiando algunos elementos significativos que condicionan la biosfera y por ende la existencia en el planeta tierra. (Milán, 2012)

Según la investigación Inserción de gestión integral de riesgo y cambio climático como eje transversal en la asignatura Evaluación de Impacto Ambiental, en la carrera de Ciencias Ambientales realizado en FAREM - Estelí se trabajó el desarrollo de habilidades en la elaboración de términos de referencia para estudios de impacto ambiental con un enfoque de gestión de riesgo y cambio climático, viendo la importancia de que los estudiantes visualicen la aplicabilidad del conocimiento a situaciones concretas de riesgo, mediante la ejecución de un plan de acción. (Mairena, Soza, \& Vilchez, 2013).

Es de suma importancia manifestar un interés natural por el estudio del riesgo climático, ante el cual la humanidad está y estará sujeta durante los próximos años, es por esto que el propósito de la investigación es valorar las percepciones socioculturales de los pobladores de la comunidad El Limón de la ciudad de Estelí, ante el un riesgo climático.

La percepción marca la pauta para la acción, y es necesaria una adaptación orientada al manejo eficiente de los recursos naturales, pues el conocimiento sobre los riesgos y su debida preparación generan una disminución substancial del riesgo climático. (PNUD, 2013)

Como trabajador social el principal interés es la parte humanística de un problema, es por ello que se dirigió la investigación al análisis de las percepciones socioculturales de los pobladores, sabiendo que influyen directamente en la manera de concebir el mundo, de actuar y tomar decisiones ante un riesgo.

Durante el proceso del estudio se desarrolló un aprendizaje significativo entre docentes y estudiantes, permitiendo lograr el análisis de las percepciones socioculturales desde una visión de autogestión ante el riesgo climático.

\section{MATERIALES Y MÉTODOS}

La investigación se realizó a partir de un diseño cualitativo con metodología de Investigación-Acción. Para fines del estudio se seleccionaron habitantes de la comunidad el Limón que se encuentran en riesgo.

La muestra estuvo conformada por 54 informantes para la encuesta, que representan el total de las viviendas de la comunidad, 3 informantes para la entrevista semiestructurada y 20 pobladores que participaron en el grupo focal. El proceso de selección para la entrevista se realizó mediante el muestreo no probabilístico, de tipo intencional, ya que se debía cumplir los siguientes criterios de selección: 1) Tener al menos 3 años de habitar en la comunidad El Limón; 2) Tener más de 18 años de edad; 3) No haber participado en la encuesta; y 4) Disponibilidad para formar parte del estudio.

Para la recolección de la información se aplicaron las técnicas de la encuesta, guía de observación, entrevista semi-estructurada, grupo focal y análisis documental. La encuesta estaba dividida en dos dimensiones, la sociocultural y la socioambiental, conteniendo más de 70 items, dentro de los cuales estaban conocimientos de los pobladores y aspectos económicos y productivos. La guía de observación elaborada en forma de lista de cotejo únicamente contenía aspectos específicos a observar. En la entrevista semi-estructurada se elaboró una guía de preguntas dirigidas con el objetivo de recopilar información complementaria a los otros instrumentos y de esta manera valorar las percepciones socioculturales. El grupo focal se realizó con el 
objetivo de trabajar las principales problemáticas de la comunidad, para determinar las posibles soluciones prácticas. El análisis documental consistió en la revisión de resultados de otros proyectos ejecutados en la comunidad para analizar el impacto logrado en la población.

Una vez obtenida la información de la encuesta, primeramente se hizo el análisis de los resultados de acurdo a cada ítem, utilizando Excel y SPSS, para hacer más fácil la comprensión. Para los resultados de la entrevista se hizo una trascripción fiel de la información, posteriormente se hizo una lectura para el análisis de acuerdo a categorías, abordado desde los objetivos.

También se hizo uso del principio de la triangulación para verificar si los datos obtenidos a través de las diferentes fuentes y técnicas de recolección de información guardaban relación o discrepaban entre sí. Además de realizar una análisis de contenido de acuerdo a su enfoque.

\section{RESULTADOS Y DISCUSIÓN}

\section{Percepciones socioculturales ante el riesgo climático}

Lo primero es que al ser una comunidad en aras de la urbanización por su posición geográfica, el rol social y la forma de vida cambia de una manera trepidante, influyendo así en las concepciones, actitudes y comportamientos de las familias y la población en general, lo que determina las percepciones como se explica en la teoría según (Lazos \& Paré Cruz, 2000), surgen no solo del análisis de la cultura sino también de la evolución social y del contexto que se desarrolla.

Uno de los principales hallazgos de este estudio es el abandono de las labores agrícolas, siendo una comunidad geográficamente rural, está influenciado en gran medida por dos situaciones concretas, una son los efectos del cambio climático en la producción agrícola y la segunda en la cercanía de la ciudad a la comunidad, lo que ha producido también un incremento significativo de la migración del campo a la ciudad por motivos laborales permitiendo recibir salarios bajos pero estables.

Lo que nos lleva a analizar que los pobladores viven una disyuntiva de ser la comunidad geográficamente una zona rural y a nivel laboral o económico sobrevivir de las actividades urbanas, como lo vemos en la figura 1. Esta situación los ubica en condiciones distantes a las problemáticas de los contextos rurales ante riesgos climáticos. Además se evidencia que no poseen suficiente conocimiento sobre cómo actuar ante un riesgo climático, lo cual los ubica en una situación aún más vulnerable.

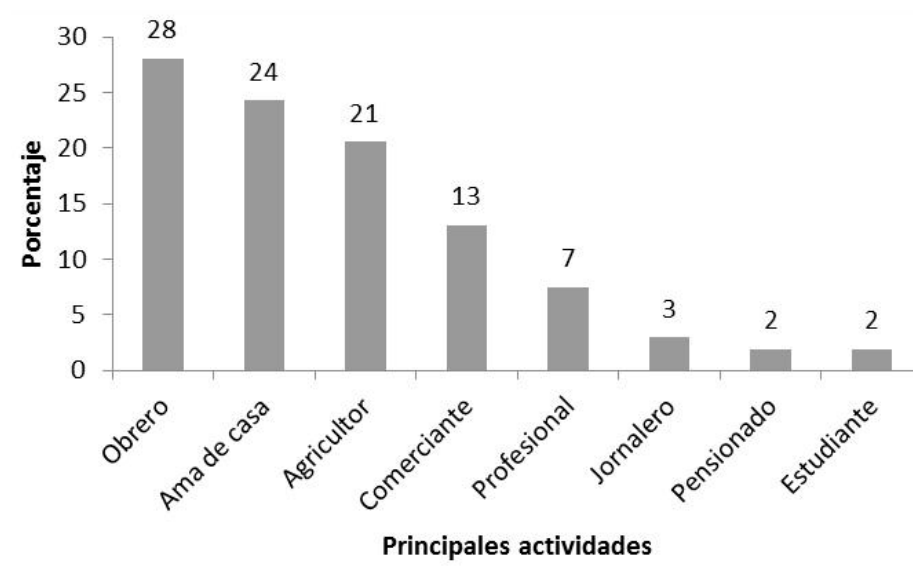

Figura 1: Principales actividades económicas a la que se dedican los pobladores de la comunidad El Limón.

\section{Impacto en las actividades socioambientales}

En lo referido al impacto de las actividades socioambientales se ve enmarcado en la baja producción de los cultivos y el aumento de plagas y enfermedades, así como en la escases del agua en la comunidad, lo cual deja entrever de igual manera la falta conocimiento científico en los pobladores, evidenciado en su forma de ver los problemas y dar una respuesta concreta a la situación que están viviendo como vemos en la figura 2. 


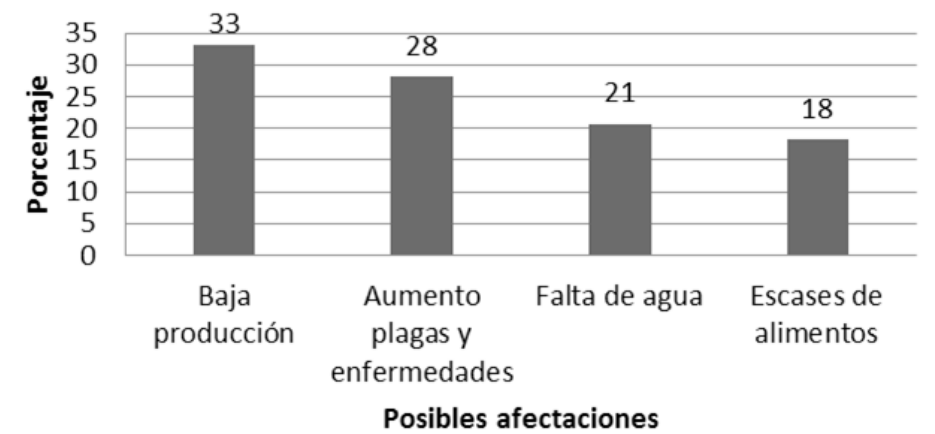

Figura 2: Impacto en las actividades socioambientales de los pobladores.

Se constató que los proyectos que han intervenido en la comunidad no han logrado impactos significativos de incidencia en la apropiación de los pobladores con información pertinente sobre que es riesgo y cambio climático. En los resultados de la encuesta se refleja que las capacitaciones recibidas únicamente se han limitado al abordaje superficial sobre los daños inmediatos de la deforestación y beneficios de la reforestación, créditos o sobre generalidades de diferentes temas referidos al desarrollo comunitario, minimizando así la importancia a la problemática de cambio climático.

\section{Preparación ante el riesgo climático}

La falta de un comité comunitario capacitado y preparado para intervenir en un eventual riesgo es uno de los hallazgos más evidentes, pues las únicas organizaciones comunitarias que se encuentran presentes son los Comités de Agua Potable, los Gabinetes de la familia, brigada de salud y cooperativa de mujeres (referido a crédito), esto deja entrever la vulnerabilidad que se tiene como comunidad, la necesidad de la creación de este comité, y la actuación para la atención a esta problemática.

En la parte agrícola (Campos, Chaput, Crespín, \& Sedó, 2010) refiere que la población rural tiene muchos insumos en cuanto a conocimiento sobre acciones de prevención y protección de fuentes de agua, suelo, cultivos, entre otras como medida de adaptación, pero en la parte social las acciones ante un riesgo climático son de poco dominio público, debido a la centralización de la participación comunitaria en los líderes y lideresas.

A partir de los resultados obtenidos se elaboró una propuesta de acción en conjunto con la comunidad como medida de adaptación al cambio climático, propiciando una autogestión comunitaria.

La propuesta metodológica contempla una actividad para compartir el plan de acción a la comunidad, así como la elección de líderes y lideresas para la formación del comité de prevención ante riesgos, el cual estaría encargado entre otras cosas del acompañamiento comunitario orientado al fortalecimiento de los conocimientos sobre riesgo, adaptación al cambio climático y jornadas de limpieza y reforestación, por medio de asambleas, visitas casa a casa y reuniones.

El plan de acción pretende fomentar la autogestión comunitaria, y que de esta manera permita que los pobladores estén preparados y se empoderen sobre lo referido a riesgo, para hacer de este una solución como medida de adaptación al cambio climático.

\section{CONCLUSIONES}

Como resultado del trabajo realizado se logró concluir que el contexto en el cual se ubica la comunidad, en referencia a su posición geográfica influye mucho en la forma de concebir el mundo, las actitudes hacia la vida y las incoherencias, pues los pobladores no poseen el suficiente conocimiento ante los riesgos climáticos, y únicamente perciben como un riesgo lo que se refiere al río que atraviesa la comunidad, esto unido a la cercanía de la ciudad hace de alguna manera que las percepciones cambien, pues su entorno se modificó de lo rural a lo urbano en cierta medida.

De igual manera el impacto que se percibe en cuanto a las actividades socioambientales es poco debido principalmente a la falta de participación comunitaria 
y a la poca información recibida, pues dicho impacto se percibe únicamente en el aumento de plagas en los cultivos y enfermedades tanto para los animales, como las mismas personas.

Al evidenciar la falta de una organización comunitaria preparada para atender riesgos, y la inexistencia de planes de contingencia en la comunidad, sabiendo que la información que posee la población es obtenida de la radio o televisión, se ve necesaria la creación de un comité encargado de atender los riesgos climáticos dentro de la comunidad, conformada por pobladores de diferentes edades y dispuestos a contribuir para el bien de su comunidad, pues la solución desde el punto de vista de los pobladores es conocer el fenómeno y adaptarse.

Es importante el analizar la necesidad de profesionales del trabajo social en el abordaje de diferentes temáticas en el área de investigación, pues los problemas acontecidos mayormente tienen que ver con el ser humano y su desarrollo social, económico, ambiental, etc. Y desde esta óptica el análisis de los aportes sociales y culturales constituye una fuente primordial en el abordaje de cualquier situación concreta y de esta manera poder determinar también las posibles soluciones o medidas de tratar estos.

\section{AGRADECIMIENTOS}

Este documento ha sido generado gracias y en el marco del proyecto 'Elementos críticos para el intercambio de conocimientos entre la Comunidad "El Limón" y la Estación Experimental para el Estudio del Trópico Seco, orientados al manejo eficiente de los recursos naturales como medida de adaptación ante el
Cambio Climático"'. (Identificador B6), del Programa Universitario para la Reducción del Riesgo de Desastres y Adaptación al Cambio Climático en Centroamérica del Consejo Superior Universitario Centroamericano (PRIDCA-CSUCA), con el auspicio de la cooperación Suiza para América Central (COSUDE) y ejecutado por la Estación Experimental para el Estudio del Trópico Seco, (FAREM Estelí / UNAN Managua) en el período julio 2014 enero 2016.

Al Programa de Reducción de la Vulnerabilidad y Adaptación al Cambio Climático en las Región de las Segovias - Nicaragua, ejecutado por MARENA y el PNUD con el Auspicio de la Cooperación Suiza para América Central (COSUDE), por el aporte financiero para realizar esta investigación.

\section{BIBLIOGRAFÍA}

Campos, M., Chaput, P., Crespín, M. T., \& Sedó, K. (2010). Adaptarnos al Cambio Climático la Unica Salida (2da ed.). Managua: EDISA.

Lazos, O., \& Paré Cruz, E. (2000). Evolucion de la percepción . Buenos Aires: Neva Luz.

Mairena Mairena, E. M., Soza Peralta, A., \& Vilchez Ponce, L. A. (2013). Inserción de gestión integral de riesgo y cambio climático como eje transversal en la asignatura Evaluación de Impacto Ambiental, en la carrera de Ciencias Ambientales, durante el primer semestre del 2013. Estelí.

Milán, J.A. (2012). Apuntes Sobre el Cambio Climatico en Nicaragua. Managua/ Nicaragua: Copy Expres. PNUD. (2013). Términos Importantes. Inventario de Prácticas y Tecnologías para la adaptación al Cambio Climático, 12. 